\title{
Soft X-ray follow-up of five hard X-ray emitters
}

\author{
L.Pavan $^{* 1}$, E. Bozzo ${ }^{1}$, C. Ferrigno1 ${ }^{1}$, M. Falanga ${ }^{2}$, S. Campana ${ }^{3}$, S. Paltani ${ }^{1}$, L. Stella ${ }^{4}$, R. \\ Walter $^{1}$ \\ ${ }^{1}$ ISDC - Universitè de Genève. \\ Chemin d'Ecogia 16, CH-1290 Versoix, Switzerland \\ ${ }^{2}$ International Space Science Institute (ISSI), Switzerland \\ ${ }^{3}$ INAF - Osservatorio Astronomico di Brera, Italy \\ ${ }^{4}$ INAF - Osservatorio Astronomico di Roma, Italy \\ E-mail: Lucia.Pavan@unige.ch
}

\begin{abstract}
We studied the soft-X-ray emission of five hard-X sources: IGR J08262-3736, IGR J17354-3255, IGR J16328-4726, SAX J1818.6-1703 and IGR J17348-2045. These sources are: a confirmed supergiant high mass X-ray binary (IGR J08262-3736); candidates (IGR J17354-3255, IGR J163284726) and confirmed (SAX J1818.6-1703) supergiant fast X-ray transients; IGR J17348-2045 is one of the as-yet unidentified objects discovered with INTEGRAL.

Thanks to dedicated XMM-Newton observations, we obtained the first detailed soft X-ray spectral and timing study of IGR J08262-3736. The results obtained from the observations of IGR J173543255 and IGR J16328-4726 provided further support in favor of their association with the class of Supergiant Fast X-ray Transients. SAX J1818.6-1703, observed close to phase 0.5, was not detected by XMM-Newton, thus supporting the idea that this source reaches its lowest X-ray luminosity ( $\left.\sim 10^{32} \mathrm{erg} / \mathrm{s}\right)$ around apastron. For IGR J17348-2045 we identified for the first time the soft X-ray counterpart and proposed the association with a close-by radio object, suggestive of an extragalactic origin. In this proceeding we discuss the results obtained from the XMM-Newton follow-up observations of all the five sources.
\end{abstract}

"An INTEGRAL view of the high-energy sky (the first 10 years)" 9th INTEGRAL Workshop and celebration of the 10th anniversary of the launch,

October 15-19, 2012

Bibliotheque Nationale de France, Paris, France

\footnotetext{
* Speaker.
} 


\section{Introduction}

High-mass X-ray binaries (HMXBs) comprise a compact object orbiting a massive O-B spectral type star. The compact object, usually a neutron star (NS), emits a conspicuous amount of $\mathrm{X}$-ray radiation (up to luminosities of $\sim 10^{37} \mathrm{erg} / \mathrm{s}$ ) due to the accretion of matter from the $\mathrm{OB}$ companion. Depending on the nature of the giant star, HMXBs are classified as Be (BeXBs) or supergiant (SGXBs) X-ray binaries. While in the first case the NS is orbiting around the companion in a highly eccentric orbit (see e.g. Stella et al. 1986); in SGXBs the compact object moves around its companion in a nearly circular orbit. The luminosity of these latter systems has in general less pronounced changes along the orbit with respect to the BeXBs, but can be subject to non-periodic variations on time scales of seconds to hours, with $\Delta L_{X} \sim 10-50$, due to hydrodynamic instabilities in the wind of the supergiant companion (Negueruela 2010). A subclass of SGXBs, the supergiant fast X-ray transients (SFXTs), spend a large fraction of their time (Romano et al. 2011) in a quiescent state $\left(\mathrm{L}_{X} \sim 10^{32}-10^{33} \mathrm{erg} / \mathrm{s}\right)$, and only sporadically undergo bright outbursts $\left(\Delta L_{X} \sim 10^{4}-10^{5}\right)$ lasting a few hours and reaching peak luminosities of $L_{X} \sim 10^{37} \mathrm{erg} / \mathrm{s}$ (Walter \& Zurita Heras 2007). The outbursts of SFXTs are associated to the accretion of particularly dense clumps as in other SGXBs, but the origin of the lower persistent luminosity and much more pronounced variability of these sources is still a matter of debate (see e.g. in 't Zand 2005; Bozzo et al. 2011, and references therein).

In this proceeding we report on the results of XMM-Newton observations of four HMXBs discovered with INTEGRAL and BeppoSAX: one classical SGXB (IGR J08262-3736), two candidates (IGR J17354-3255, IGR J16328-4726) and one confirmed (SAX J1818.6-1703) SFXTs. We also report on the XMM-Newton observation of the still unclassified INTEGRAL source IGR J173482045. A detailed description of the performed analysis can be found in Bozzo et al. (2012).

\section{Results}

We used all the available XMM-Newton observations of the five sources obtained through the guest observation programs awarded to our research group. XMM-Newton observation data files (ODFs) were processed using the standard XMM-Newton Science Analysis System (v. 11.0) Lightcurves were background-subtracted and barycenter-corrected; spectra were background subtracted, rebinned, and where required barycenter-corrected as well.

\subsection{IGR J08262-3736}

IGR J08262-3736 is associated to the OB-V star SS 188, at a distance of $6.1 \mathrm{kpc}$ (Malizia et al. 2011). Its X-ray spectrum could be fit with a simple power-law model $\left(N_{\mathrm{H}}=1.5 \times 10^{22} \mathrm{~cm}^{-2}\right.$, photon index $\Gamma=2$ ). The X-ray luminosity of the source was $\sim 2.4 \times 10^{33} \mathrm{erg} / \mathrm{s}$ (Malizia et al. 2011).

We obtained an XMM-Newton observation of IGR J08262-3736 on 2010 October 16 for a total exposure time of $\sim 25 \mathrm{ks}$, with all three EPIC cameras operated in full frame mode. During this observation, the source displayed a moderate variability, with two relatively small flares occurring about $1.5 \times 10^{4} \mathrm{~s}$ after the beginning of the observation. The hardness ratio of the source (see Fig. 1) does not show clear variations in the source spectral properties with the count-rate. The Epic-pn spectrum of the source can not be fit by a simple powerlaw model, and requires either an additional 

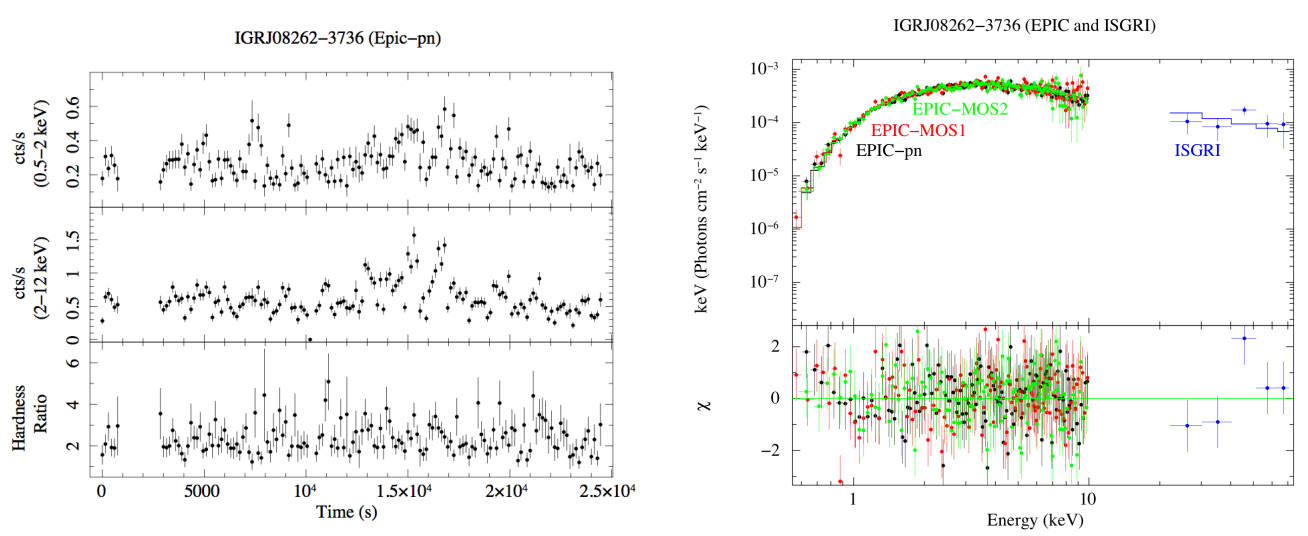

Figure 1: Left: XMM-Newton lightcurve of IGR J08262-3736 in the 0.5-2 keV and 2-12 keV energy bands and the corresponding hardness ratio. Right: Average Epic-pn and INTEGRAL/ISGRI spectrum of IGR J08262-3736. The bestfit model (solid line) is obtained with a partially covered power-law model. The residuals from the fit are shown in the bottom panel.

blackbody component at low energies, or the introduction of a partial covering (see Fig. 1). We also analyzed the mean spectrum obtained from the long-term monitoring of the source with ISGRI (obtained through the HEAVENS online tool ${ }^{1}$ ). The XMM and INTEGRAL spectra can be fitted simultaneously, by means of the same model described above, showing that the source has a virtually constant persistent X-ray flux. These timing and spectral behavior are typical of classical SGXBs. The presence of "soft excess" in the spectrum is believed to be an ubiquitous feature of binary systems hosting accreting NSs (Hickox et al. 2004). In wind accreting binaries with Xray luminosities comparable to that of IGR J08262-3736 $\left(\simeq 3 \times 10^{34} \mathrm{erg} / \mathrm{s}\right.$, assuming a distance of $6.1 \mathrm{kpc}$ ), the soft component is most likely originated by thermal X-ray photons close to the NS surface or by photoionized or collisionally heated diffuse gas in the binary system. As an alternative interpretation, also a partial-covering model would provide an acceptable description of the X-ray spectrum. In this case, the soft excess would be produced by the effect of partial obscuration of the emission from the NS by the surrounding high-density material (see e.g., Tomsick et al. 2009b).

\subsection{IGR J17354-3255}

IGR J17354-3255 was discovered with INTEGRAL in 2006 (Kuulkers et al. 2006). Long term monitoring of the source carried out with the INTEGRAL/ISGRI and Swift/BAT showed that it is a weak persistent emitter in the hard X-rays (average 18-60 keV flux of $1.1 \mathrm{mCrab}$ ), displaying only sporadically flares with duration from few hours to $\sim 1$ day. This behavior, together with a periodic modulation detected in the hard X-ray data at nearly 8.45 days, led to the interpretation of IGR J17354-3255 as a HMXBs, possibly a SFXT (D'Aì et al. 2011; Sguera et al. 2011). In the soft X-ray domain, IGR J17354-3255 was observed with Swift/XRT and Chandra, showing two sources within its INTEGRAL error circle (S1 and S2, Vercellone et al. 2009). A more pronounced variability of S1 (=CXOU J173527.5-325554) led to the conclusion that this source is the most likely counterpart to IGR J17354-3255 (see e.g. Tomsick et al. 2009a).

\footnotetext{
${ }^{1}$ http://www.isdc.unige.ch/heavens/
} 
XMM-Newton observed the region of IGR J17354-3255 on 2011 March 6 for a total effective exposure time of $20 \mathrm{ks}$. MOS1 and pn cameras were operated in full-frame, while MOS2 was in small-window mode. Among the two candidate soft X-ray counterparts of IGR J17354-3255, in our XMM observation only S2 was clearly detected, at a flux level of $2.4 \times 10^{-13} \mathrm{erg} \mathrm{cm}^{-2} \mathrm{~s}^{-1}$ in the 0.5-10 keV range, compatible with previous measurements (Vercellone et al. 2009). No significant evidence for coherent modulations was found in the Epic data, thus supporting the idea that this is a persistent object unrelated with the INTEGRAL source. We could obtain a $3 \sigma$ upperlimit for S1 of $7 \times 10^{14} \mathrm{erg} \mathrm{cm}^{-2} \mathrm{~s}^{-1}$, a factor of $\sim 6$ lower than previous estimations. The increased dynamic range of S1/IGR J17354-3255 gives strength to the SFXT interpretation of the source.

\subsection{IGR J16328-4726}

Corbet et al. (2010) found an orbital period of $\sim 10 \mathrm{~d}$ from the hard X-ray transient INTEGRAL source IGR J16328-4726, hinting towards an interpretation as HMXB, possibly a supergiant system. INTEGRAL/ISGRI detected two outbursts lasting a few hours (Fiocchi et al. 2010) from this source. On both occasions the ISGRI spectrum could be fit with a simple power-law model ( $\simeq \simeq 2$ 2.6) with a flux of $2-3.3 \times 10^{-10} \mathrm{erg} \mathrm{cm}^{2} \mathrm{~s}^{-1}(20-50 \mathrm{keV})$. The source was not detected at quiescent level, and the $3 \sigma$ upper limit showed that IGR J16328-4726 has a dynamical range of at least 100 . The fast flaring behavior of the source, and its spectral properties, suggested that IGR J16328-4726 is a further member of the SFXT class discovered with INTEGRAL.

IGR J16328-4726 was observed by XMM-Newton on 2011 February 20 for a total effective exposure time of 14.7 (21) ks for the Epic-pn (MOS) cameras. The source displayed a clear variability, with relatively small flares $\left(\Delta \mathrm{L}_{\mathrm{X}} \lesssim 10\right)$ occurring during periods of low-level $\mathrm{X}$-ray activity (Fig. 2). These flares closely resemble the ones observed from the SFXT prototypes (Bozzo et al. 2010), and therefore are in further support to the association of IGR J16328-4726 with this class of objects. The average Epic-pn spectrum extracted during the observation is well described by a strongly absorbed power-law model $\left(N_{\mathrm{H}} \sim 17.5 \times 10^{22} \mathrm{~cm}^{-2}, \Gamma=1.5 \pm 0.1\right.$; see Fig. 2$)$. The flux ranges from $6.4 \times 10^{-12}$ (quiescent level) up to $5 \times 10^{-11} \mathrm{erg} \mathrm{cm}^{-2} \mathrm{~s}^{-1}$. At odds with other SFXTs observed in quiescence, we do not detect any soft excess in the X-ray spectrum of the source. This spectral component might have gone undetected in IGR J16328-4726 due to its relatively high absorption column density and the lower exposure time with respect to XMM-Newton observations of other quiescent SFXT sources with similar X-ray luminosity (Bozzo et al. 2010).

\subsection{SAX J1818.6-1703}

SAX J1818.6-1703 is a confirmed SFXT source, discovered by BeppoSAX on 1998 during a 2-hours period of intense X-ray activity (in 't Zand et al. 1998). Since then, several outbursts from the source have been recorded with INTEGRAL and Swift (see e.g. Sidoli et al. 2009). The source is at distance of $2.5 \mathrm{kpc}$, and has an orbital period of $30 \pm 0.1 \mathrm{~d}$ (Bird et al. 2009). Most of the observed outbursts took place when the NS is close to the periastron (Zurita Heras \& Chaty 2009), however, outbursts in several periastron passages were missing. This behavior might be explained by assuming that the NS in SAXJ1818.6-1703 has a highly eccentric orbit and is sporadically accreting mass from the clumpy wind of its supergiant companion. The eccentricity of 0.3-0.4 inferred for SAX J1818.6-1703 however can hardly support this scenario (see e.g. Bird et al. 2009). 

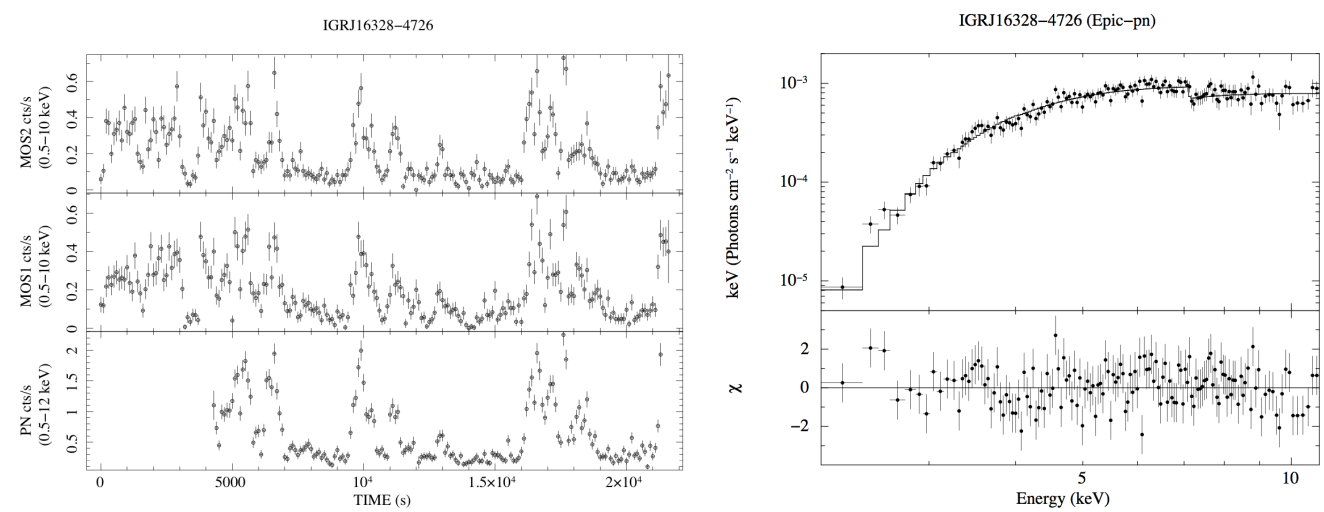

Figure 2: Left:XMM-Newton lightcurve of IGR J16328-4726 in the $0.5-12 \mathrm{keV}$ and $0.5-10 \mathrm{keV}$ energy range for the Epic-pn and Epic-MOS, respectively. The time bin is in all cases $100 \mathrm{~s}$. Right: XMM/pn spectrum of IGR J16328-4726. The residuals from a fit with absorbed powerlaw model are shown in the bottom panel.

We obtained an XMM-Newton observation of the region around SAX J1818.6-1703 on 2010 March 21 for a total exposure time of $45 \mathrm{ks}$, at orbital phase $0.53 \pm 0.18$ - close to apastron (with ephemeris given by Bird et al. 2009). The Epic-pn camera was operated in Full Frame mode, while the MOS1 and MOS2 were operated in Small Window and Fast Uncompressed mode, respectively. SAX J1818.6-1703 was not detected with the Epic cameras. We estimated from these data a 3- $\sigma$ upper limit on the source luminosity of $\sim 2 \times 10^{32} \mathrm{erg} / \mathrm{s}$, comparable with the one estimated by the previous non-detection of the source at phase 0.51 (Bozzo et al. 2008). The mechanism involved in the prolonged quiescent state of SAX J1818.6-1703 is not known yet. Proposed models include inhibition of accretion by the NS, due to centrifugal and/or magnetic barrier (Bozzo et al. 2008); or alternatively accretion from a highly rarefied and structured companion wind (see, e.g. Zurita Heras \& Chaty 2009, and references therein).

\subsection{IGR J17348-2045}

IGR J17348-2045 is an unclassified INTEGRAL source Bird et al. (2009). The estimated fluxes in the $20-40 \mathrm{keV}$ and $40-100 \mathrm{keV}$ energy bands are $0.3 \pm 0.1 \mathrm{mCrab}$ and $0.9 \pm 0.1 \mathrm{mCrab}$, respectively.

The source was observed by XMM-Newton on 2011 March 2 for a total effective exposure time of 10 (4) ksec for Epic-MOS (pn). The three EPIC detectors were operated in Full Frame mode. Inside the INTEGRAL error circle we detect two soft X-ray sources (named XMMU J173458.8204530 and XMMU J173449.2-204244, according to the XMM-Newton convention). The former source is brighter and located close to the center of the INTEGRAL error-circle; the latter is too dim to extract any meaningful spectral information (see Fig. 3). We assume in the following that XMMU J173458.8-204530 is the true counterpart to IGR J17348-2045. Its XMM spectrum is modeled as a strongly absorbed PL $\left(\mathrm{N}_{H} \sim 10^{23} \mathrm{~cm}^{-2} ; \Gamma=1.5\right)$. A simultaneous fit with the longterm ISGRI spectrum (obtained from HEAVENS) is compatible with the source being a persistent hard X-ray emitter. The uncertainty in the Epic-MOS position does not allow a firm identification of counterparts at other wavelengths, but we remark here the possible association with the radio source NVSS J173459-204533. If this association will be confirmed by future observations, we 


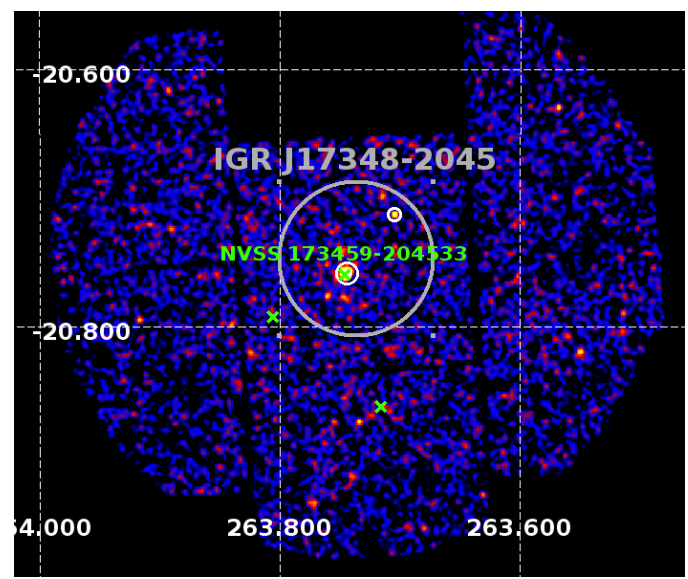

Figure 3: Epic/MOS image of IGR J17348-2045 in the $0.5-10 \mathrm{keV}$ band. The two soft X-ray counterparts of IGR J17348-2045 detected are indicated as white circles. The radio object NVSS J173459-204533, spatially coincident with XMMU J173458.8-204530, is also indicated in green.

argue that IGR J17348-2045 could be one of the highly absorbed active Galactic nuclei (AGN) discovered with INTEGRAL (see e.g. Ricci et al. 2011, and references therein).

\section{Conclusions}

We analyzed in the 1-10 keV region five hard X-ray sources, through dedicated XMM-Newton observations (published in Bozzo et al. 2012). The observations provided the first detailed spectral and timing study of IGR J08262-3736, showing signatures of an accreting neutron star. The variability of the 1-10 keV counterpart to IGR J17354-3255, assessed through the deepest upperlimit of the source, favors its interpretation as SFXT. A similar interpretation is strengthened for IGR J16328-4726 thanks to the observed time variability. The mechanism involved in the prolonged quiescent state of SAX J1818.6-1703, and SFXTs in general, is still matter of debate. Thanks to the first 1-10 keV study of IGR J17348-2045 we could identify its soft X-ray emission, and localize a possible radio counterpart. We suggest for this source an extragalactic origin.

\section{References}

Bird, A. J., et al. 2009, MNRAS, 393, L11

Bozzo, E., et al. 2008, ApJ, 683, 1031

Bozzo, E., et al. 2011, A\&A, 531, A130

Bozzo, E., et al. 2012, A\&A, 544

Bozzo, E., et al. 2010, A\&A, 519, A6

Corbet, R. H. D., et al. 2010, Astr. Tel., 2588

D'Aì, A., et al. 2011, A\&A, 529, A30

Fiocchi, M., et al. 2010, ApJl, 725, L68

Hickox, R. C., et al. 2004, ApJ, 614, 881

in 't Zand, J., et al. 1998, IAUcirc, 6840, 2

in 't Zand, J. 2005, A\&A, 441, L1

Kreykenbohm, et al. 2008, A\&A, 492, 511

Kuulkers, E., et al. 2006, Astr. Tel., 874
Malizia, A., et al. 2011, Astr. Tel., 3294

Negueruela, I. 2010, ASP Conf. Series, 422, 57

Ricci, C., et al. 2011, A\&A, 532, A102

Romano, P., et al. 2011, MNRAS, 410, 1825

Sguera, V., et al. 2006, ApJ, 646, 452

Sguera, V., et al. 2011, MNRAS, 417, 573

Sidoli, L., et al. 2009, MNRAS, 400, 258

Stella, L., et al. 1986, ApJ, 308, 669

Tomsick, J. A., et al. ApJ, 701, 811

Tomsick, J. A., et al. 2009b, ApJ, 694, 344

Torrejón, J. M., et al. 2010, A\&A, 510, A61

Vercellone, S., et al. 2009, Astr. Tel., 2019

Walter, R. \& Zurita Heras, J. 2007, A\&A, 476, 335

Zurita Heras, J. A. \& Chaty, S. 2009, A\&A, 493, L1 